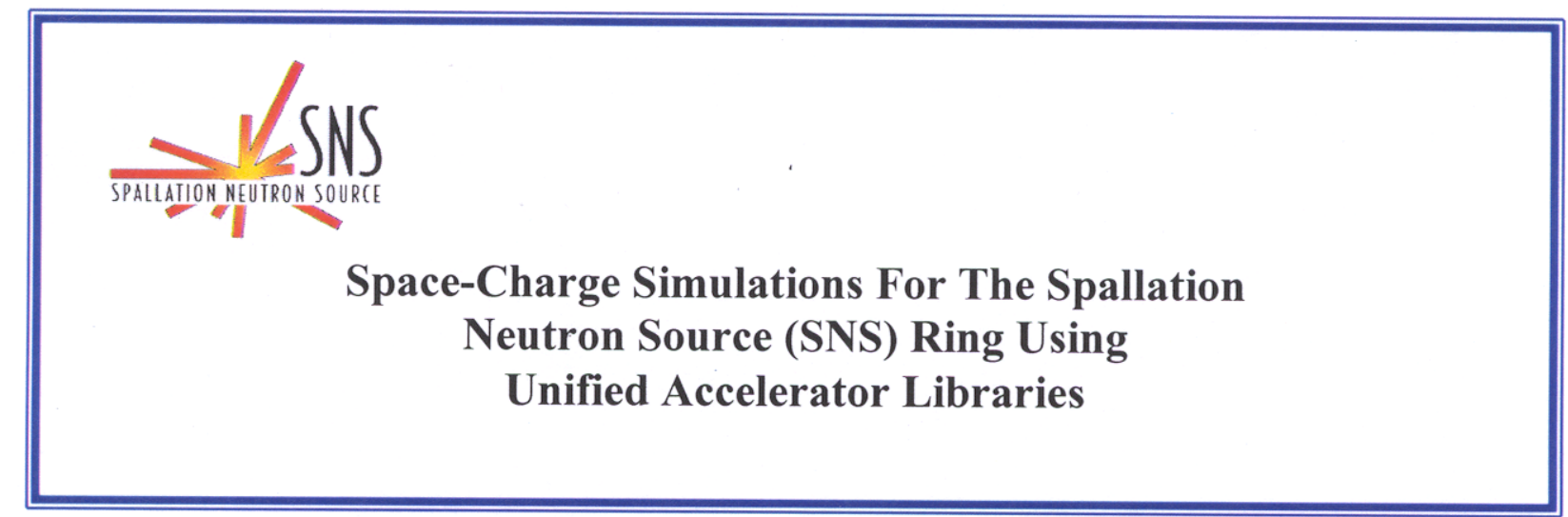

BNL/SNS TECHNICAL NOTE

NO. 086

A.V. Fedotov, N. Malitsky, J. Wei

January 25,2001

COLLIDER-ACCELERATOR DEPARTMENT BROOKHAVEN NATIONAL LABORATORY UPTON, NEW YORK 11973 


\title{
SPACE-CHARGE SIMULATIONS FOR THE SPALLATION NEUTRON SOURCE (SNS) RING USING UNIFIED ACCELERATOR LIBRARIES
}

\author{
A.V. Fedotov, N. Malitsky, and J. Wei \\ C-A Department, BNL, Bldg. 817, Upton, NY 11973-5000
}

(January 25, 2001)

\begin{abstract}
The latest designs for high-intensity proton rings require minimal beam loss. Although the tune depression in the ring is very small compared with highintensity linacs, space-charge contributions to beam loss through halo formation may be significant. The goal of achieving low-level beam loss becomes especially challenging in the presence of both the space charge and magnet imperfections. This puts very high expectations on the code used for computer simulations. The Spallation Neutron Source (SNS) ring dynamics presents a complex combination of several physical effects and dynamic processes. Some of them, such as field errors and misalignments are supported in general-purpose accelerator codes. Other effects, such as space charge, are actual only for high intensity hadron rings and distributed into a set of independent specialized programs. A new SNS package, based on Unified Accelerator Libraries (UAL) environment, addresses all these effects together. This paper describes some basic features of the SNS simulation environment and demonstrates its application for the space-charge simulations in the SNS accumulator ring.
\end{abstract}




\section{INTRODUCTION}

Space charge is a fundamental limitation in high-intensity circular accelerators. In the SNS, which pushes beyond existing intensity levels, space-charge effects will be especially important. The importance of space charge and halo formation in high-intensity linacs has been widely recognized (see, for example, extensive literature in [1]; also some new developments were recently reported [2]). In rings, however, an understanding of these issues appears to be even more important: for economic reasons, in a linac one can attempt to accept the halo, while in a ring one must try to avoid halo formation because of a relatively small beam pipe acceptance / beam size ratio. A discussion of halo formation issues in circular accelerators was recently presented [3]. The "parametric halo" (usually cited in linac community as "the halo") is just a special case of a halo caused by the parametric resonance. Such parametric resonance mechanism is not necessarily the dominant one for halo formation in high-intensity rings [3]. In general, a halo can be produced by various mechanisms [4]. Some of such mechanisms which could give significant contribution to halo formation in the SNS accumulator ring were recently summarized [5].

The SNS ring beam dynamics is a complex combination of several physical effects. Because of such a complexity analytic estimates of halo formation are very limited. Hence, realistic computer simulations are required. Most of our previous space-charge simulations were done with the ORBIT [6] and SIMPSONS [7], which helped us to get a general picture of the space-charge dynamics in the SNS ring $[5,8,9]$. To understand the importance of required simulations we recall that beam power of the proposed SNS ring is an order of magnitude above that of existing accelerator facilities. This imposes extremely strict requirement on uncontrolled beam loss at $10^{-4}$ level [10], such level is not yet achieved in existing proton synchrotrons and accumulator rings. In order to address such low-level losses

we should closely reproduce all complex physics of a realistic machine. The environment of Unified Accelerator Libraries (UAL) [11] suits to such purposes. Thus, a new SNS package was developed, using the UAL [12]. This package allows us to study beam dynamics includ- 
ing such effects as injection painting, magnet field errors and misalignments, magnet fringe fields, space charge, etc. This paper gives a general description of the package and its first application to the SNS space-charge studies.

\section{SNS RING SIMULATION ENVIRONMENT}

\section{A. Unified Accelerator Libraries}

The UAL are designed as a customizable and extendible environment for developing diverse accelerator applications. Its main architectural principle is a separation of physical entities and mathematical abstractions from algorithms. The accelerator algorithms are implemented as classes that share data via Common Accelerator Objects (Element, Bunch, Twiss, etc.). This highly flexible structure has facilitated selecting and implementing more appropriate software design pattern and accelerator approaches, supporting project-specific requirements. At this time, the UAL joins six object-oriented programs (see Fig. 1): PAC (Platform for Accelerator Codes) [13], TEAPOT (Thin Element Program for Optics and Tracking) [14], ZLIB (Numerical Library for Differential Algebra ) [15], ACCSIM (Accumulator Simulation Code) [16], ORBIT (Objective Ring Beam Injection and Tracking Code) [6], and ALE (Accelerator Library Extensions). The Application Programming Interface (API), written in Perl, provides a universal shell for integrating and managing all project extensions. Such UAL environment has already been successfully applied to LHC [17] and RHIC [18]. For the SNS ring applications, we have developed the SNS-specific package [12] that binds together all these components and allows one to model the multi-turn injection including various physical effects.

\section{B. SNS package}

The SNS ring simulation environment employs the same Facade design pattern used in other UAL applications. According to this pattern, applications are based on the additional 
Perl layer that provides the project-specific shell to the underlying UAL $\mathrm{C}++$ and Perl components. The overall architecture of the SNS environment is illustrated in Fig. 2. A physicist interacts with the SNS::Shell interface that encompasses a set of high-level MADlike or TEAPOT-like commands, such as use, analysis, hsteer, and others. Most of these commands are project-independent, and inherited from the basis class ALE::Shell. The multi-turn injection algorithms are considered as an extension of the present UAL environment and implemented directly in the SNS::Shell class.

To facilitate the study of a complex scenario, we have implemented the injection framework with three basis classes (See Fig. 3): SNS::PaintingScheme, SNS::Integrator, and SNS::Diagnostics. Each basis class originates an open collection of alternative approaches that can be selected in any combination with the instances of two other collections. For example, the following script (see Fig. 4) invokes the correlated painting (SNS::CorrelatedScheme) with the matrix-based integrator (SNS::Mapper) and default diagnostics (SNS::DefaultDiagnostics). Usually, the matrix-based integrator is effective for the initial evaluation of the new painting scenario. After accomplishing this step, one may proceed to study the impact of other effects (such as the space charge and/or magnet errors). In our framework, this transition can be done by redefinition of a single Perl reference.

At this time, the SNS package includes four integrators: SNS::Mapper, SNS::Tracker, SNS::SCMapper, and SNS::SCTracker. Each integrator is implemented as an adapter to the corresponding UAL modules. The SNS::Mapper is based on the ZLIB Truncated Power Series (TPS). The SNS::Tracker keeps a pointer to the TEAPOT integrator that deals with non-linear dynamics, magnet errors, and misalignments. The SNS::SCMapper is identical to the ORBIT approach and uses linear matrices for ring elements and thin lenses for the space-charge effects. The fourth class, the SNS::SCTracker, is a special case of the SNS integrators because it dispatches the request to two UAL modules, TEAPOT and ORBIT. It allows one to extend capabilities of both approaches and to apply them to new problems. 


\section{Numerical approaches}

Some of the important features which were implemented, benchmarked, and used in the SNS package are: injection painting, magnet fringe fields, magnet non-linearities and space charge.

\section{Injection painting.}

During the multi-turn injection into the SNS ring, beam is painted over a large phase space volume in order to reduce the space-charge tune shift and to minimize the number of traversals through the stripping foil. The development of beam halo depends strongly on the choice of painting scheme. Prevention of such halo is crucial for satisfying low level beam loss requirements [5,9]. Implementation of this dynamical process is based on the ACCSIM approach [16]. However, the control of different scenarios is programmed directly with the Perl Application Programming Interface (API) that provides simple access to the UAL packages.

\section{Fringe fields.}

Since the aperture of the ring magnets is comparable to magnet lengths, fringe field impact is very important [20]. The fringe fields are included through the Taylor maps extracted from the fringe field models. Both realistic three-dimensional (3-D) fringe field

calculations with the MARYLIE [19] and "hard-edge" approximation showed importance of the longitudinal field derivatives, and produced very similar behavior [20]. Currently, we are using maps (up to 5th order) based on both the "hard-edge" formulas and the realistic 3-D fields. 


\section{Single-particle dynamics and non-linearities.}

Special characteristics of the SNS ring are large beam emittances (up to $240 \pi \mathrm{mm}$ $\mathrm{mrad})$, and large beam pipe apertures. Not surprisingly, this brings a variety of non-linear effects which are a direct consequence of large particle amplitudes. Such non-linearities can shift particles in undesired directions, dramatically decreasing the dynamic aperture. The study and understanding of these effects thus becomes very important. The simulation of non-linear magnet fields and misalignments were done with the TEAPOT [14] approach. TEAPOT approximates magnet elements by thin multipoles but treats the non-linear equation of motion exactly. To be confident in our non-linear dynamics studies, the UAL was benchmarked against MARYLIE 3.0 [19], which is a thick element code with an approximate

treatment of equations of the motion. Some results of this benchmarking were presented in [22]. Figure 5 shows excellent agreement between these two codes for the tune shift due to the kinematic non-linearity, which arises from high order terms proportional to the transverse momenta $p_{x}, p_{y}$ in the expansion of the standard square-root relativistic Hamiltonian [22]. This plot is generated by launching particles in five different transverse directions with the amplitudes going up to $480 \pi \mathrm{mm}$ mrad (neither non-linear elements nor magnet errors are present in the lattice). Data obtained with the MARYLIE is presented by color circles, while UAL's data is given by white dots inside the color circles.

\section{Multi-particle dynamics with the space charge.}

The space-charge effect has the largest impact on beam dynamics and halo growth in the SNS accumulator ring, and has to be included in the common model. It is currently implemented through the ORBIT space-charge module, detailed description of which could be found, for example, in $[8,21]$. Briefly, this module is based on a Particle-in-cell (PIC) method employing a bilinear distribution of macroparticles to the nodes of rectangular grid with subsequent use of fast-Fourier-transform method to approximate the full non-linear 
space-charge force. This module was successfully benchmarked vs code SIMPSONS [9], and vs experimental measurements [21].

For the space-charge studies we developed two independent integrators, SCMapper and SCTracker. The SCMapper, which adopts linear matrices approach for ring element treatment, was extensively used to ensure perfect agreement between the UAL and ORBIT codes. The SCTracker contains the ORBIT space-charge algorithm but treats ring elements based on the TEAPOT approach which allows to include magnet errors in consistent manner. Similar to a single-particle dynamics, it includes all non-linear features of the motion even in the absence of magnet errors.

In this paper we will use numerical examples based on correlated painting which results in a square shape beam desired by the target requirements [10]. These studies refer to the hybrid lattice (FODO arcs and doublet straights) of the 220 meter circumference SNS ring. In Figs. 6 and 7 we present 2-D density plots (X-Y) for resulting beam distribution based on the square-root bump function without and with the space charge, respectively. Simulations were done with the SCMapper without magnet errors. The inclusion of the space charge leads to a rapid azimuthal diffusion with some spreading in the radial direction. For this case the 2-D beam densities, based on simulations, agree well with analytic predictions. Exact treatment of non-linear motion (without magnet errors) via the SCTracker package leads to an additional spreading along the diagonals. The comparison between two approaches is shown in Figs. 8 and 9 for the SCMapper and SCTracker, respectively. The difference between the number of particles in the halo, observed in Figs. 8 - 9, becomes important when we want to predict particle loss at a very low level, which is our ultimate goal [10].

\section{Computational efficiency}

The requirement of beam loss predictions at $10^{-3}-10^{-4}$ level forces us to use around $10^{5}$ macro-particles in our simulations, to obtain good statistics. Up to this point our priority was to include all necessary physics in simulations rather than use large number of particles 
but with simplified physics. The standard compromise between speed of calculation and physics is not quite applicable to our case since the requirement on beam losses an order of magnitude smaller than the one achieved in existing high-intensity machine. We thus need to keep all required physics in order to produce credible predictions of beam loss. At this point we note that the speed of integrator becomes very slow with most of the physics correctly included. The typical run for $50 \mathrm{~K}$ particles with the full-injection scenario, space charge, fringe fields and magnet errors takes about 50 hours of 1 CPU time on Sun station. This resulted in our decision to deploy the UAL on the parallel cluster. Parallel version of the UAL was recently successfully developed and we now proceed with the simulations on the parallel cluster [23]. The detailed description of computational efficiency on parallel cluster will be reported in the nearest future.

\section{SIMULATIONS WITH THE SPACE CHARGE}

\section{A. Space-charge induced resonances}

One of the advantages of the SNS lattice is that horizontal and vertical tunes can be both adjusted by more than one unit without significant optical mismatch [10]. This provides us with the flexibility of the choice of a working point. However, investigation of the proposed working points requires systematic studies since each of the working points has its advantages and disadvantages [10]. It also requires a detailed study of various resonances and their correction.

Magnet field errors can drive particles into machine resonances, when betatron tunes have resonant values. The effect of space charge is typically treated as a perturbation which results in the shift of a betatron tune. This usually sets criterion to have the incoherent space-charge tune shift smaller than the distance (in the tune space) to the nearest low-order resonance lines. Such criterion turns out to be too restrictive. A correct treatment also needs to take into account collective beam oscillations as was first demonstrated in [24,25]. The 
new criterion can be described in terms of "coherent" [26,27] or "effective" [5] tune shift, which makes the actual space-charge limit less restrictive, and thus helps to find the most suitable working points.

In addition to machine resonances there are also space-charge induced resonances. Here we refer to space-charge induced resonances as those which are driven by the space-charge potential rather than by the field potential of magnets. Their importance was first shown by B. Montague [28], for the dominant coupling resonance. This resonance can occur even for a linear lattice without any perturbations since it requires only zeros harmonic of Fourier component of the density perturbation. The Montague resonance is a well known mechanism which results in a typical recommendation of about one unit tune split for the high-intensity machines. For the SNS, with the base line working point $\left(Q_{x}, Q_{y}\right)=(5.82,5.8)$, this nonlinear space-charge resonance $2 Q_{x}-2 Q_{y}=0$ was also observed in numerical simulations with the full-intensity beams $[8,29,30]$ and multi-turn injection $[30,9]$. Due to the fact that this resonance is a difference resonance such coupling could lead to a significant effect only for a beam with unequal emittances. However, in the SNS case, even with equal emittance, this leads to an excessive halo for the case of correlated painting. This shifted our attention to exploration of other working points. Also, other space-charge induced resonances are possible when the driving force comes from the space-charge potential while harmonic $\mathrm{N}$ results from the magnet imperfections [31-33], or is related to the ring superperiodicty, which, in the latter case, leads to the space-charge induced superstructure resonances [31].

\section{B. Effect of magnet errors}

Working points with tune split of half-integer or more help to avoid strong coupling caused by the space-charge forces and systematic magnet errors. We thus continued our investigation of working points with the splitting of tunes by a half-unit $\left(Q_{x}, Q_{y}\right)=(6.3,5.8)$. This allowed us to preserve the desired beam shape obtained via correlated painting, which is shown, for example, in Figs. 6 - 7 at the end of 1200 multi-turn injection. The next step, 
of course, is to understand how this beam distribution changes in the presence of magnet errors. Figure 10 shows the effect of various errors for this working point. Here we plot the percentage of particles in the halo outside total emittance $\epsilon_{x}+\epsilon_{y}[\mathrm{~mm} \mathrm{mrad}]$, when we paint to a square shape beam with the emittance of $120 \pi[\mathrm{mm} \mathrm{mrad}]$ in each of the transverse directions. One can see that the effect of expected [10] systematic and random field errors is negligible. An additional effect of small random magnet misalignments of $0.1 \mathrm{~mm}$ in both horizontal and vertical direction is very small. The effect of a small tilt of $0.2 \mathrm{mrad}$ in the bending magnets was also found to be not important. The situation dramatically changes when we introduce relatively small tilt $(0.2 \mathrm{mrad})$ in the quadrupoles. This effect is shown in Fig. 11 for the beam tail, and in Fig. 12 for the beam profile. In the absence of the space-charge, with the same tilt error in the quadrupoles, beam spreading disappears as demonstrated in Fig. 13. After detailed study of such resonant behavior we confirmed that this is the manifestation of the sum resonance $Q_{x}+Q_{y}=12$. It takes place due to the combined effect of the space charge and skew-quadrupole errors. In the absence of the space charge the strength of introduced skew-quadrupole component (tilt of $0.2 \mathrm{mrad}$ ) was not sufficient for particles to be trapped into the resonance which requires $\Delta Q$ of 0.1 . However, the space charge depresses the tunes, and some particles are trapped into this sum resonance even for a relatively small skew-quadrupole components. Note that observed resonance is not the space-charge induced resonance since, in this case, it is driven by the skew-quadrupole field, and the role of the space charge is to push particles into the resonance. To confirm the nature of this resonance the detailed study of the width of the resonance was conducted by examining the strength of introduced tilts and the phase advance between the quadrupoles. Eventually, all the observed features of this resonance were also reproduced without the space charge by introducing required strength of the skew-quadrupole components, and exciting the correspondent harmonic based on the formula for $Q_{x}+Q_{y}=p$ resonance [34]:

$$
\Delta Q=\frac{1}{4 \pi R} \int_{0}^{2 \pi} \sqrt{\beta_{x} \beta_{y}} K e^{i\left[\mu_{x}+\mu_{y}-\left(Q_{x}+Q_{y}-p\right) \theta\right]} d \theta,
$$


where $K$ is a skew-quadrupole coefficient, $Q_{x}$ and $Q_{y}$ are horizontal and vertical tunes, and $\mu_{x}, \mu_{y}$ are phase advances in horizontal and vertical planes, respectively. Using this formula, we confirmed the excitation of the sum resonance for various combination of the tilt strength and phase advances. Such resonance can be corrected using the decoupling schemes. However, due to a very large space-charge tune shift we will need very precise correction of the skew-quadrupole terms to eliminate beam blow-up shown in Fig. 14. Slight detuning of the working point could also help, as demonstrated in Fig. 15. Unfortunately, such detuning becomes dangerous (especially in the direction of tune decreasing) since we can now cross other resonances excited by magnet imperfections. Despite the possibility of correction, the vicinity of this dominant sum resonance makes our working point $\left(Q_{x}, Q_{y}\right)=(6.3,5.8)$ less attractive, as in the case of the Montague resonance. One can see that finding the best choice

of working point becomes very challenging for the SNS due to its special characteristics of a very large tune spread mainly associated with the space charge, chromaticity and magnet fringe fields. It requires systematic approach of applying various correction schemes first, and then searching for the optimum tunes by exploring the tune space and beam parameters. We expect that the UAL will be an important tool in addressing these questions following our demonstration of its capability to consider all these important effects.

\section{SUMMARY}

The SNS ring dynamics presents a complex combination of several physical effects and dynamic processes. Its special characteristics of a very large tune spread, associated with the space charge, chromaticity and magnet fringe fields, impose very challenging task of avoiding machine and space-charge induced resonances. The necessity to achieve and predict extremely low level beam losses thus put very strict requirement on computer simulations. To address these issues of the SNS project, the new computer package was developed based on the UAL environment. This paper presents description of the package, and demonstrates its application to space-charge simulations in the presence of magnet errors. As an example, 
we presented an impact of various magnet errors on beam distribution for the base working point of the SNS. We also discussed the resonant behavior for this working point which takes place due to the combined effect of the linear coupling and space-charge tune spread.

\section{ACKNOWLEDGMENTS}

We would like to thank M. Blaskiewicz for numerous useful discussions, D.T. Abell for helping to benchmark single-particle dynamics and implementation of fringe field maps, J. Holmes for his help with diagnostic package. We also thank J. Beebe-Wang, N. Catalan-

Lasheras, J. Galambos, R.L. Gluckstern, Y.Y. Lee, Y. Papaphilippou and the rest of the SNS team for useful suggestions and discussions. 


\section{REFERENCES}

[1] R.L. Gluckstern and A.V. Fedotov, Workshop on beam halo and scraping, (Wisconsin, 1999), Fermilab Conference Proceedings 00-185, p. 22 (2000).

[2] S. Strasburg and R.C. Davidson, Phys. Rev. E, V. 61, 5, p. 5753 (2000).

[3] A.V. Fedotov, R.L. Gluckstern, and M. Venturini, as in Ref. [1], p. 27 (2000).

[4] Beam Halo Working Group Summary, as in Ref. [1], p. 21 (2000).

[5] A.V. Fedotov, D.T. Abell, J. Beebe-Wang, Y.Y. Lee, N. Malitsky, J. Wei, and R.L. Gluckstern, Proceedings of EPAC 2000, Vienna, Austria, p. 1289 (2000).

[6] J.D. Galambos, J.A. Holmes, D.K. Olsen, A. Luccio, and J. Beebe-Wang, ORBIT Users Manual, SNS/ORNL/AP Tech. Note 011 (1999).

[7] S. Machida and M. Ikegami, AIP Conference Proceeding 448, edited by A.U. Luccio and W.T. Weng (AIP N.Y. 1998), p. 73 (1998).

[8] J.A. Holmes, V.V. Danilov, J.D. Galambos, D. Jeon, and D.K. Olsen, PRST AB, Vol. 2, 114202 (1999); and references there in.

[9] J. Beebe-Wang, A.V. Fedotov, J. Wei, and S. Machida, Proceedings of EPAC 2000, Vienna, Austria, p. 1286 (2000); and references there in.

[10] J.Wei et al., PRST AB, V. 3, 080101 (2000).

[11] N. Malitsky and R. Talman, AIP Proceedings 391, p. 337 (1996).

[12] N. Malitsky, J. Smith, J. Wei, and R. Talman, Proceedings of PAC 1999 (New York, USA), p. 2713 (1999).

[13] N. Malitsky, A. Reshetov and G. Bourianoff, SSC Technical Report, SSCL-675 (1994).

[14] L. Schachinger and R. Talman, Particle Accel., Vol. 22, p.35 (1987).

[15] Y. Yan and C-Y. Yan, SSC Technical Report, SSCL-300 (1990). 
[16] F.W. Jones, "Developments in the ACCSIM Multi-particle Tracking and Simulation Code", Proceedings of PAC 1997, Vancouver, Canada, p. 2597 (1997).

[17] W. Fischer, F. Pilat and V. Ptitsin, Proceedings of PAC 1999, New York, USA, p. 2716 (1999).

[18] F. Pilat, S. Tepikian, C.G. Trahera and N. Malitsky, Technical Report, RHIC-AP-146 (1996).

[19] A.J. Dragt, D.R. Douglas, F. Neri, E. Forest, L.M. Healy, P. Schutt, and J. van Zeijts, MARYLIE 3.0 Users Manual, University of Maryland, Physics Department Report (1999).

[20] Y. Papaphilippou and D.T. Abell, Proceedings of EPAC 2000, Vienna, Austria, p. 1453 (2000).

[21] J.D. Galambos, S. Danilov, D. Jeon, J.A. Holmes, D.K. Olsen, F. Neri, and M. Plum, PRST AB, V. 3, 034201 (2000).

[22] A.V. Fedotov, D.T. Abell, Y.Y. Lee, N. Malitsky, Y. Papaphilippou, and J. Wei, Proceedings of EPAC 2000, Vienna, Austria, p. 1492 (2000).

[23] A. Shishlo, private communications.

[24] L. Smith, Proceedings of the International Conference in High Energy Accelerators, Dubna, Russia, 1963, p. 1232 (1964).

[25] F. Sacherer, Ph.D. thesis, University of California at Berkeley (Lawrence Radiation Laboratory Report No. UCRL-18454), 1968.

[26] I. Hofmann, Phys. Rev. E57, p. 4713 (1998).

[27] R. Baartman, AIP Conference Proceeding 448, edited by A.U. Luccio and W.T. Weng (AIP, N.Y. 1998), p.56. 
[28] B.W. Montague, CERN Report 68-38 (1968).

[29] D. Jeon, J.A. Holmes, V.V. Danilov, J.D. Galambos, and D.K. Olsen, Phys. Rev. E, V. 60, 6, p. 7479 (1999).

[30] A.V. Fedotov, internal report (unpublished); also presented as a talk at Wisconsin Workshop (Ref. [1]), September 1999.

[31] S. Machida, Nucl. Inst. Methods, A309, p. 43 (1991); A384, p. 316 (1997).

[32] Y. Shoji and H. Sato, KEK Preprint 97-38 (1997).

[33] I. Hofmann and K. Beckert, IEEE Trans. of Nucl. Science, Vol. NS-32, N. 5, p. 2264 (1985).

[34] G. Guignard, CERN Report 75-23 (1975). 


\section{FIGURES}

\begin{tabular}{|c|c|c|c|c|c|}
\hline \multicolumn{2}{|c|}{ ALE } & \multicolumn{4}{|c|}{ Application Scripts } \\
\hline \begin{tabular}{|l|}
$D A$ \\
Integrators \\
\end{tabular} & $\begin{array}{l}\text { User } \\
\text { Interface } \\
\end{array}$ & RHIC & CESR & LHC & SNS \\
\hline \multicolumn{6}{|c|}{ PERL API } \\
\hline$\stackrel{\vartheta}{\exists}$ & 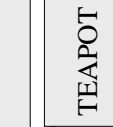 & 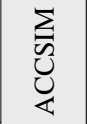 & 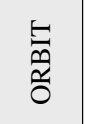 & & 馬 \\
\hline
\end{tabular}

FIG. 1. Unified Accelerators Libraries (UAL) environment.

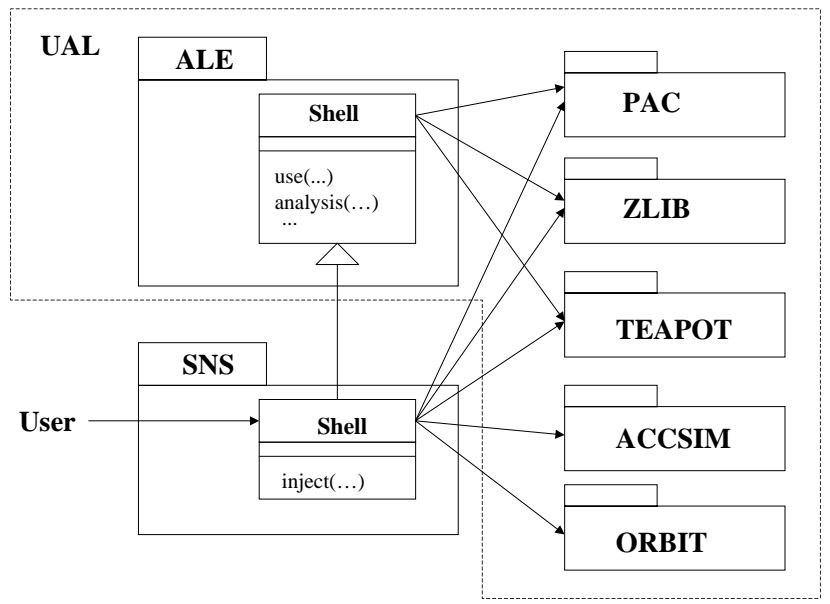

FIG. 2. SNS simulation environment. 


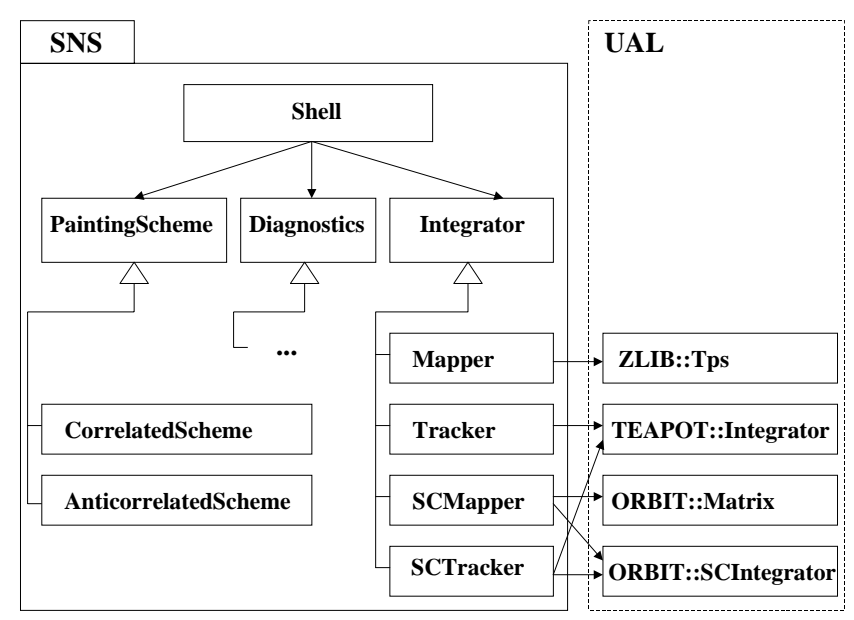

FIG. 3. Structure of the SNS package.

my \$shell = new SNS::Shell( $\ldots)$;

my \$integrator $=$ new SNS::Mapper(...);

my \$scheme - new SNS::CorrelatedScheme (...);

my $\$$ diagnostics = new SNS::DefaultDiagnostics $(. .$.$) ;$

$\cdots$

\$shell->inject("integrator" => \$integrator,

"scheme" => \$scheme,

"diagnostics" => \$diagnostics);

FIG. 4. Part of user's script for the SNS injection simulation (written in Perl).

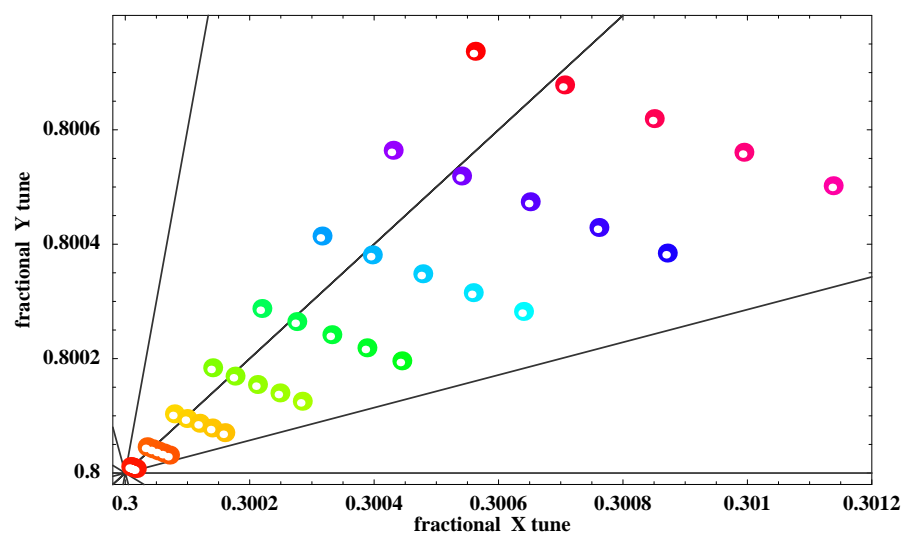


FIG. 5. Kinematic non-linearity X-Y tune foot-print. Particles are launched in five different transverse directions with the amplitudes going up to $480 \pi \mathrm{mm}$ mrad. Data obtained with MARYLIE is presented by color circles, while UAL's data is given by white dots inside the color circles.

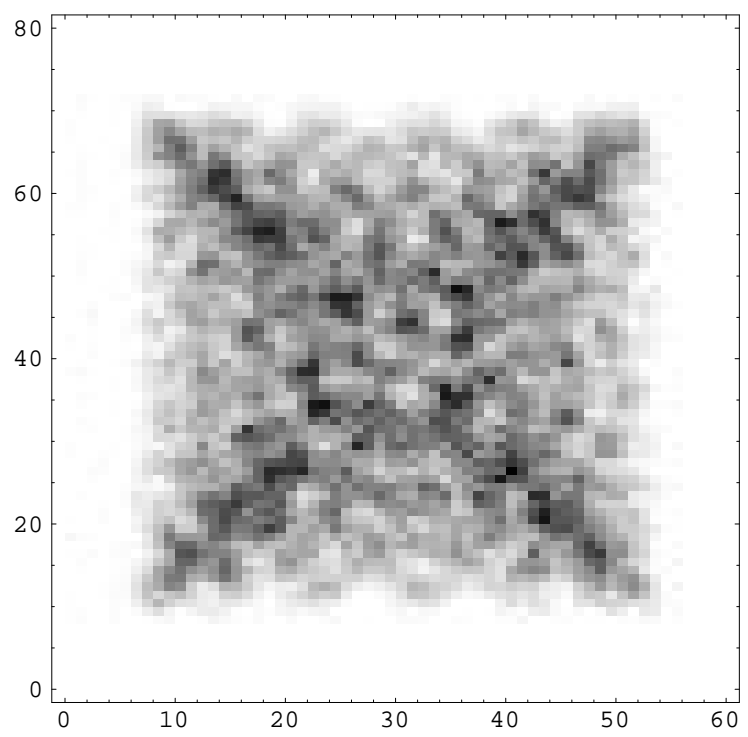

FIG. 6. 2-D density plot (X-Y) for correlated painting with the square-root bump function (without the space charge), using SCMapper/ORBIT algorithm.

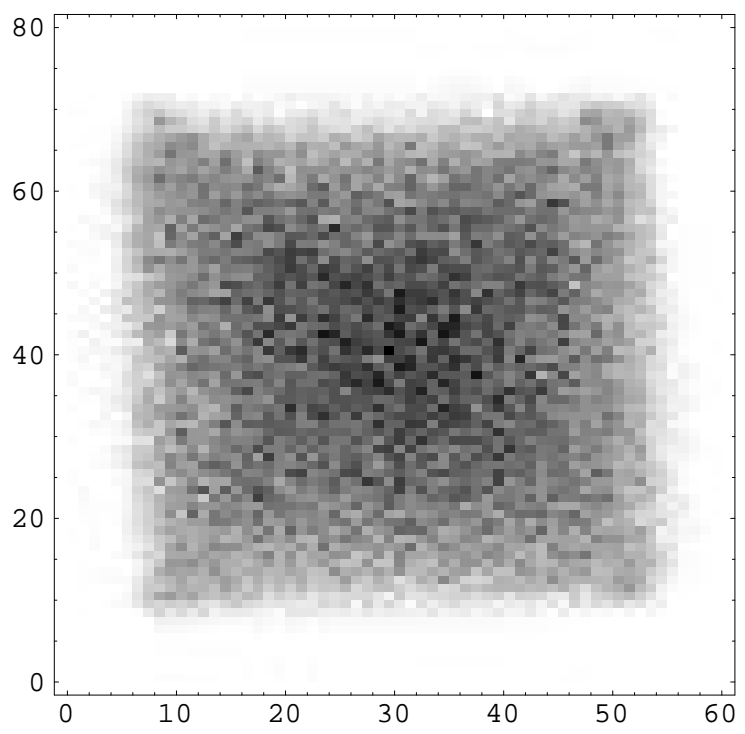

FIG. 7. 2-D density plot (X-Y) for correlated painting with the square-root bump function (with the space charge), using SCMapper/ORBIT algorithm. 




FIG. 8. Beam profile (X-Y) for correlated painting, using SCMapper (ORBIT) approach with the space charge being the only source of non-linearity.

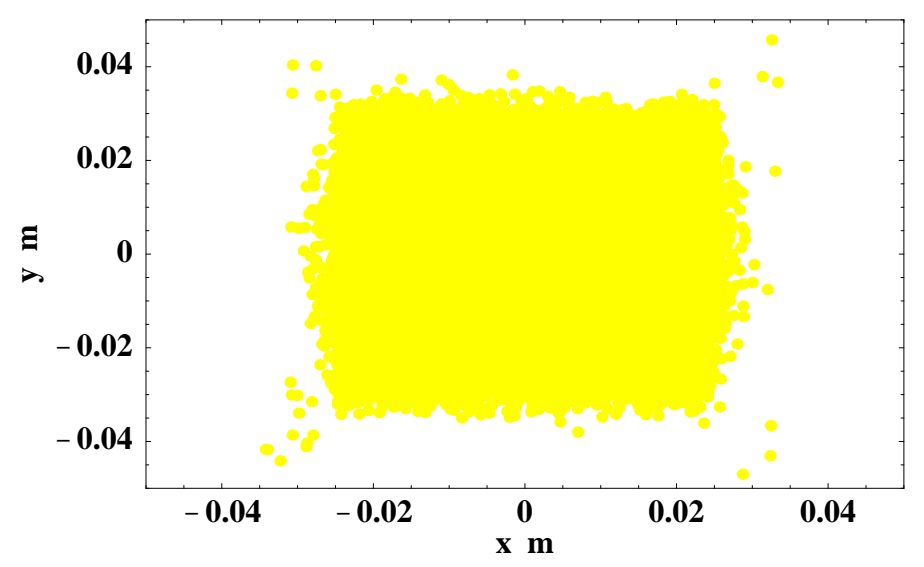

FIG. 9. Beam profile (X-Y) for correlated painting, using SCTracker (TEAPOT + ORBIT) approach which includes both the space charge and exact treatment of non-linear equations of motion.

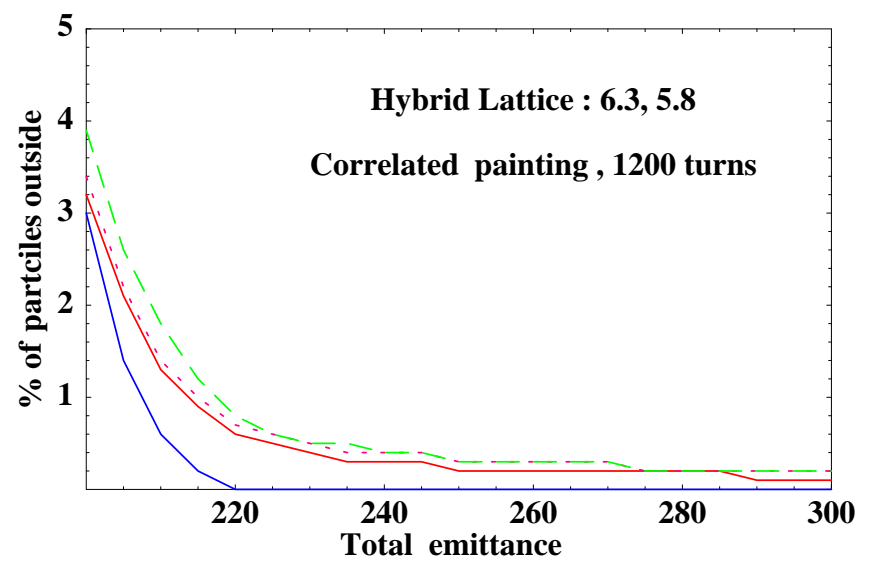


FIG. 10. Beam tail distribution for various sources of errors: 1) blue solid line (bottom) corresponds to the case without the space charge and without errors, 2) red solid line shows beam spreading due to the space charge, 3) red dotted line shows combined effect of the space charge and expected systematic and random field errors, 4) green dashed line shows an additional effect of $x, y$ misalignments of $0.1 \mathrm{~mm}$.

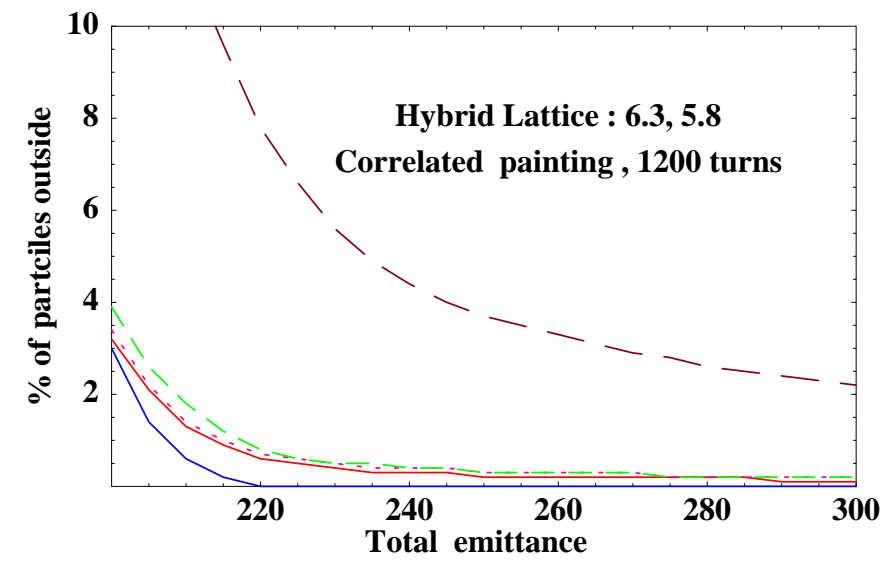

FIG. 11. Effect of quadrupole tilt on beam tail distribution. Brown dashed line (top) shows the effect of quadrupole tilt of 0.2 mrad compared to the other effects, described in Fig. 6 .

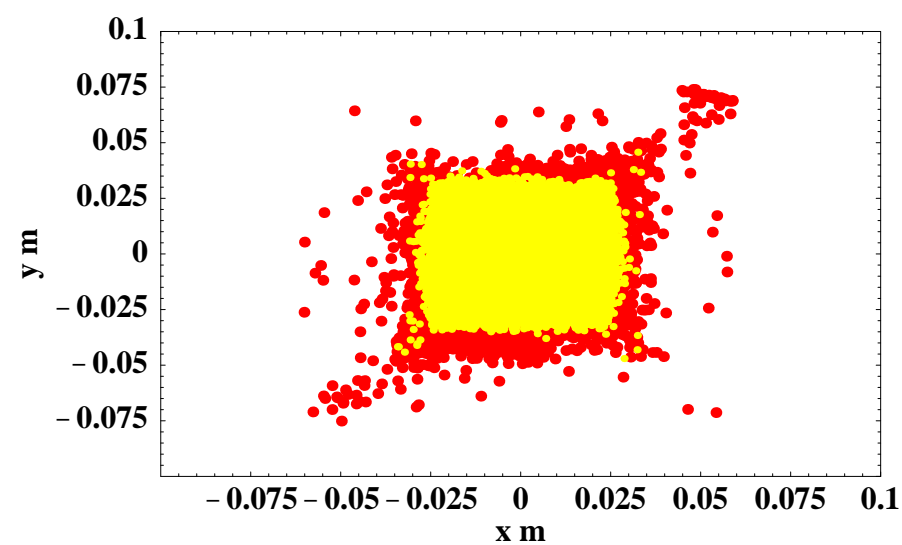

FIG. 12. Effect of quadrupole tilt on beam profile: 1) yellow color (in the middle) shows beam profile with the space charge in the absence of errors, 2) red color shows beam profile with the space charge and introduced combination of random quadrupole tilts $(0.2 \mathrm{mrad})$ which leads to the resonance. 


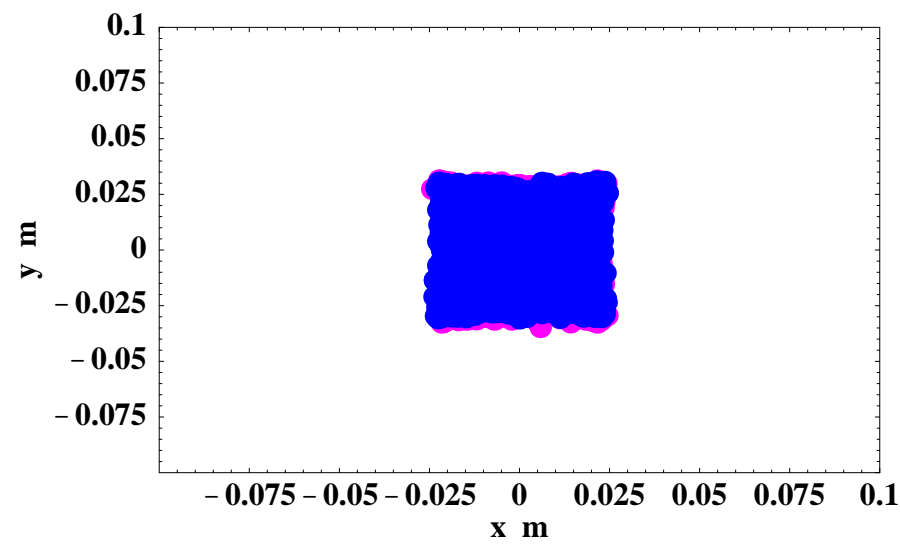

FIG. 13. Effect of quadrupole tilt in the absence of space charge: 1) blue color - no errors, no space charge, 2) pink color - quadrupole tilt (0.2 mrad), no space charge.

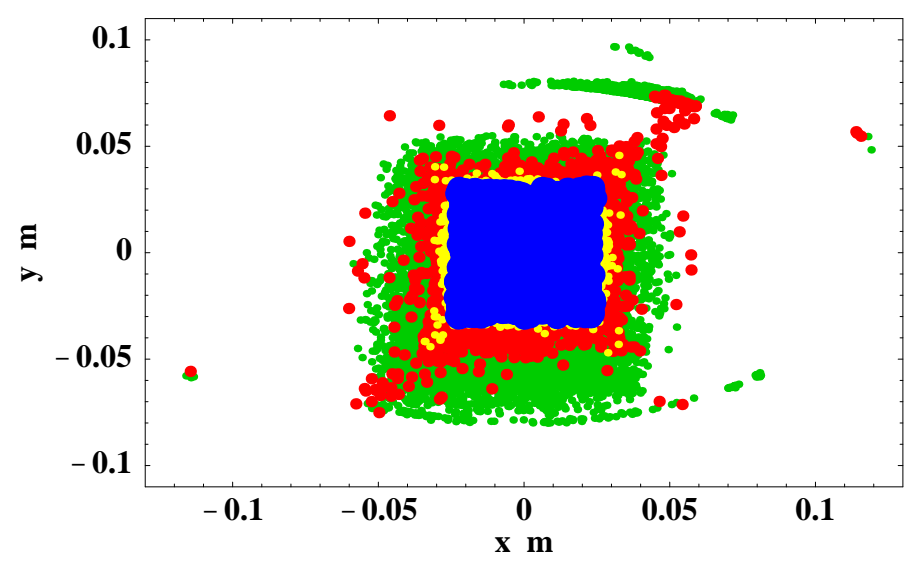

FIG. 14. Beam blow-up due to the strong skew-quadrupole sum resonance in the presence of space charge: 1) blue (in the middle) - no space charge, no errors, 2) yellow - space charge, no errors, 3) red - space charge, expected errors and quadrupole tilt (0.2 mrad), 4) green - space charge, expected errors and quadrupole tilt $(1 \mathrm{mrad})$.

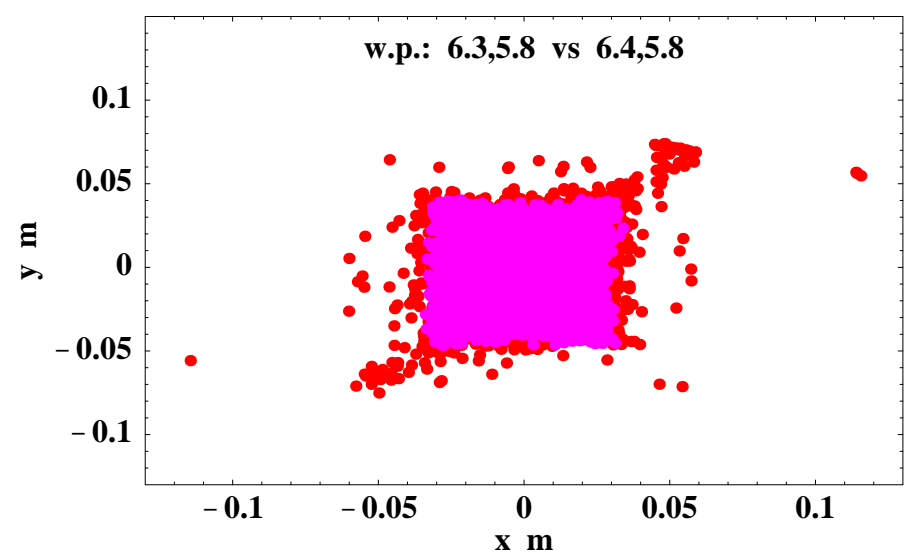


FIG. 15. Detuning of the working point to avoid skew quadrupole resonance. Both plots are given for the same distribution of random tilt errors in quadrupoles: 1) red - $\left(Q_{x}, Q_{y}\right)=(6.3,5.8)$, 2) pink (in the middle) - $\left(Q_{x}, Q_{y}\right)=(6.4,5.8)$. 